\title{
Exact top Yukawa corrections to Higgs boson decay into bottom quarks
}

\author{
Amedeo Primo* \\ Department of Physics, University of Zürich, CH-8057 Zürich, Switzerland \\ Gianmarco Sasso \\ Università di Napoli Federico II, Complesso di Monte Sant'Angelo, \\ Edificio 6 via Cintia I-80126, Napoli, Italy \\ Gábor Somogyi \\ MTA-DE Particle Physics Research Group, University of Debrecen, \\ H-4010 Debrecen, P.O. Box 105, Hungary \\ Francesco Tramontano ${ }^{\S}$ \\ Università di Napoli Federico II and INFN sezione di Napoli, Complesso di Monte Sant'Angelo, \\ Edificio 6 via Cintia I-80126, Napoli, Italy
}

(Received 30 December 2018; published 19 March 2019)

\begin{abstract}
In this paper we present the results of the exact computation of contributions to the Higgs boson decay into bottom quarks that are proportional to the top Yukawa coupling. Our computation demonstrates that approximate results already available in the literature turn out to be particularly accurate for the three physical mass values of the Higgs boson, the bottom and top quarks. Furthermore, contrary to expectations, the impact of these corrections on differential distributions relevant for the searches of the Higgs boson decaying into bottom quarks at the Large Hadron Collider is rather small.
\end{abstract}

DOI: $10.1103 /$ PhysRevD.99.054013

\section{INTRODUCTION}

The discovery of the Higgs boson [1,2] by the ATLAS [3] and CMS [4] experiments at CERN has ushered in a new era in particle physics phenomenology. The Standard Model (SM) of elementary particles is now complete and, apart from some intriguing discrepancies such as the $(g-2)_{\mu}$ anomaly or the anomalies in rare semileptonic $B$ decays (for recent reviews, see e.g., $[5,6]$ ), the observed decays and scattering phenomena at low energies do not deviate significantly from what is predicted by the SM. Still, we know that the SM cannot be the ultimate theory, if not for the lack of consistency with mainly cosmological observations, but for the fact that it contains quite a large number of parameters, which makes it unreasonable to think of it as a fundamental theory. The LHC is guiding the

\footnotetext{
*aprimo@physik.uhz.ch

sasso.gianmarco@gmail.com

*gabor.somogyi@cern.ch

francesco.tramontano@unina.it
}

Published by the American Physical Society under the terms of the Creative Commons Attribution 4.0 International license. Further distribution of this work must maintain attribution to the author(s) and the published article's title, journal citation, and DOI. Funded by SCOAP ${ }^{3}$. experimental community toward the study of the Higgs potential and the Higgs boson direct couplings with all the other particles, an experimentally previously completely unexplored sector of the SM Lagrangian. The SM makes very precise predictions for all the vertices and couplings of the Higgs boson, and the verification of these predictions is among the fundamental questions addressed at the LHC.

In particular, the gluon fusion production mechanism has given direct access to Higgs boson decay into vector bosons and an indirect access to the top Yukawa coupling. More recently, also the direct coupling of the Higgs boson to the top quark has been observed $[7,8]$. Measurements of the Higgs coupling to the tau lepton have been extracted by combining all production modes $[9,10]$, while the direct coupling to the bottom quark has been observed by exploiting the features of the $V H(V=W$ or $Z)$ associated production mechanism $[11,12]$. The decay to bottom quarks is quite special, because it is the one with the largest branching ratio. The decay width of the Higgs boson into bottom quarks has been computed at $\mathcal{O}\left(\alpha_{s}^{4}\right)$ in QCD [13-20] using an approximated treatment of the bottom quark mass, up to the first order for the electroweak corrections [21,22] and also including mixed QCD-electroweak effects [23,24]. The exact bottom mass corrections have been computed up to $\mathcal{O}\left(\alpha_{s}^{2}\right)$ [25]. A relatively large component of the two loop computation is represented by the diagrams in which the 
Higgs boson couples to a top quark loop, see Figs. 1 and 2. These two sets of diagrams are separately both UV and IR finite. However, their contributions to the total decay width have been computed only approximately in [18], finding a very compact formula that should be considered valid for values of the masses such that $m_{b} \ll m_{H} \ll m_{t}$. Comparing this formula with the rest of the two loop contributions, it turns out that these terms, which are proportional to the top Yukawa coupling $y_{t}$, account for about $30 \%$ of the total $\alpha_{s}^{2}$ contribution.

The aim of this paper is twofold. First, we wish to compute the full analytic result for the contributions to the Higgs boson decay into bottom quarks that are proportional to the top Yukawa coupling, by retaining the exact dependence on the top and bottom quark masses. This allows us to assess the impact of the neglected terms in the expansion of [18], that in principle could be of the order of $\left(m_{H} / m_{t}\right)^{4} \gtrsim 20 \%$ (see Eq. (3) in [18]). Despite the expansion in ratios of masses in [18], it could be argued that $m_{H}$ should be compared to $2 m_{t}$, rather than to $m_{t}$ itself (see Eq. (10) in [26]), yielding an order of magnitude estimate of $\left(m_{H} / 2 m_{t}\right)^{4} \sim 2 \%$ for these terms. However, the actual magnitude of the corrections is obviously influenced also by the size of the expansion coefficient. If this coefficient is $\mathcal{O}(1)$, but somewhat larger than one, the full correction may be estimated to reach the $\sim 10 \%$ level even with this counting.

Furthermore, contributions that have a modest impact on the total rate may become relevant once differential quantities are considered. Indeed, two groups have recently computed the fully differential decay width of the Higgs boson into bottom quarks up to $\mathcal{O}\left(\alpha_{s}^{2}\right)$ for the massless case, and merged this computation to the $\mathcal{O}\left(\alpha_{s}^{2}\right)$ corrections to the associated production in hadronic collisions $[27,28]$. Although the next to next to leading order correction to the
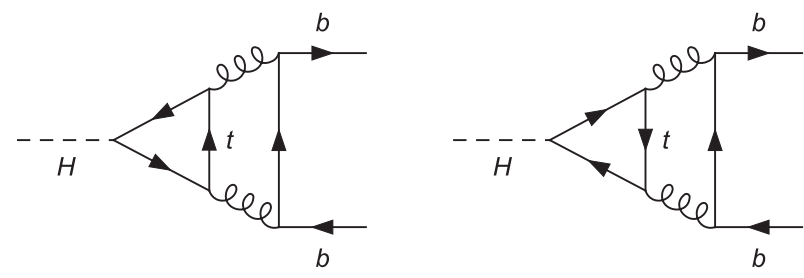

FIG. 1. Virtual $\mathcal{O}\left(\alpha_{s}^{2} y_{t}\right)$ contributions to $H \rightarrow b \bar{b}$ decay.

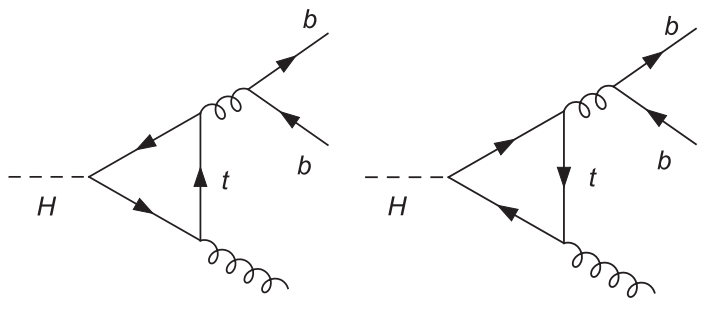

FIG. 2. Real-virtual $y_{t}$ contributions to $H \rightarrow b \bar{b}$ decay. total $H \rightarrow b \bar{b}$ decay rate is moderate, the corrections to key distributions like the transverse momentum and the mass spectra of the Higgs boson (reconstructed using the two hardest $b$-jets in the final state) are found to be very large. $y_{t}$ contributions to the Higgs decay into bottom quarks are not included in the differential results mentioned above and, therefore, it is natural to ask what the impact of these corrections is (see for example [28]). We answer this second question by presenting differential results, which include the $y_{t}$ contributions to the decay and retain the full mass dependence on the top and bottom quark masses.

Since our computation is exact in the masses of both quarks, our result is applicable also beyond the validity region $\left(m_{b} \ll m_{H} \ll m_{t}\right)$ of the approximate expression of [18], e.g., for the case of a heavy scalar coupling to the top quark.

Furthermore, the master integrals relevant for evaluating the two loop, three mass triangles with two internal masses, which we compute here for the first time, will also contribute to the Higgs decay into fermions other than the bottom quark, e.g., $H \rightarrow \mu \bar{\mu}, H \rightarrow \tau \bar{\tau}$ and $H \rightarrow c \bar{c}$. These will be the subject of further investigations.

\section{CALCULATION}

\section{A. Double virtual}

The decay $H(q) \rightarrow b\left(p_{1}\right)+\bar{b}\left(p_{2}\right)$ receives $\mathcal{O}\left(\alpha_{s}^{2} y_{t}\right)$ contributions from the interference $\left|\mathcal{M}_{y_{t}, b \bar{b}}\right|^{2}$ of the Born amplitude with two loop virtual corrections that involve a closed top quark loop,

$$
\left|\mathcal{M}_{y_{t}, b \bar{b}}\right|^{2} \equiv 2 \operatorname{Re} \mathcal{M}_{b \bar{b}}^{(0) \dagger} \mathcal{M}_{y_{t}, b \bar{b}}^{(2)},
$$

where $\mathcal{M}_{y_{t}, b \bar{b}}^{(2)}$ is given by the two diagrams shown in Fig. 1. By evaluating the Feynman diagrams, we decomposed $\left|\mathcal{M}_{y_{t}, b \bar{b}}\right|^{2}$ as

$\left|\mathcal{M}_{y_{t}, b \bar{b}}\right|^{2}=\alpha_{s}^{2} y_{t} C_{A} C_{F} \sum_{\vec{a}} c_{a_{1} \ldots a_{7}}\left(\epsilon, m_{i}^{2}\right) I_{a_{1} \ldots a_{7}}\left(\epsilon, m_{i}^{2}\right)$,

with $a_{j} \in \mathbb{Z}$ and $i=t, b, H$. In Eq. (2), the $c_{a_{1} \cdots a_{7}}$ are rational coefficients and $I_{a_{1} \ldots a_{7}}$ are two loop integrals of the type

$$
I_{a_{1} \ldots a_{7}}\left(\epsilon, m_{i}^{2}\right)=\int \frac{\mathrm{d}^{d} k_{1}}{(2 \pi)^{d}} \frac{\mathrm{d}^{d} k_{2}}{(2 \pi)^{d}} \frac{D_{7}^{a_{7}}}{D_{1}^{a_{1}} D_{2}^{a_{2}} D_{3}^{a_{3}} D_{4}^{a_{4}} D_{5}^{a_{5}} D_{6}^{a_{6}}},
$$

defined by the set of inverse propagators:

$$
\begin{aligned}
& D_{1}=k_{1}^{2}-m_{t}^{2}, \quad D_{2}=k_{2}^{2}, \quad D_{3}=\left(k_{1}-k_{2}\right)^{2}-m_{t}^{2}, \\
& D_{4}=\left(k_{1}+q\right)^{2}-m_{t}^{2}, \quad D_{5}=\left(k_{2}+q\right)^{2}, \\
& D_{6}=\left(k_{2}+q-p_{1}\right)^{2}-m_{b}^{2} \quad D_{7}=\left(k_{1}+q-p_{1}\right)^{2},
\end{aligned}
$$

with kinematics $m_{H}^{2}=q^{2}=\left(p_{1}+p_{2}\right)^{2}, p_{1}^{2}=p_{2}^{2}=m_{b}^{2}$. 
We computed the loop integrals through the consolidated differential equations (DEs) method [29-32]. First, we used integration-by-parts identities (IBPs) [33-35], generated with the help of REDUZE2 [36], in order to reduce the integrals that appear in $\left|\mathcal{M}_{y_{t}, b \bar{b}}\right|^{2}$ to a set of 20 independent master integrals (MIs) $\overrightarrow{\mathcal{I}}=\left(\mathcal{I}_{I}, \ldots, \mathcal{I}_{20}\right)$. Subsequently, we derived the analytic expression of the MIs by solving the system of coupled first-order DEs in the kinematic ratios $m_{H}^{2} / m_{t}^{2}$ and $m_{b}^{2} / m_{t}^{2}$. The structure of the DEs, and hence of their solutions, is simplified by parametrizing such ratios in terms of the variables $x$ and $y$, defined by

$$
\frac{m_{H}^{2}}{m_{t}^{2}}=-\frac{\left(1-x^{2}\right)^{2}}{x^{2}}, \quad \frac{m_{b}^{2}}{m_{t}^{2}}=\frac{\left(1-x^{2}\right)^{2}}{(1-y)^{2}} \frac{y^{2}}{x^{2}},
$$

and by using the Magnus exponential method [37-42] in order to identify a basis of MIs that fulfill a system of canonical DEs [43],

$$
\mathrm{d} \overrightarrow{\mathcal{I}}=\epsilon \mathrm{d} A \overrightarrow{\mathcal{I}}, \quad \text { with } \quad \mathrm{d} f=\sum_{z=x, y} \mathrm{~d} z \frac{\partial}{\partial z} f
$$

with $\epsilon=(4-d) / 2$. In Eq. (6), the coefficient matrix $\mathrm{d} \mathbb{A}$ is a dlog-form that contains 12 distinct letters,

$$
\begin{aligned}
\mathrm{d} A= & \mathbb{M}_{1} \operatorname{dlog}(x)+\mathbb{M}_{2} \operatorname{dlog}(1+x)+\mathbb{M}_{3} \operatorname{dlog}(1-x) \\
& +\mathbb{M}_{4} \operatorname{dlog}\left(1+x^{2}\right)+\mathbb{M}_{5} \operatorname{dlog}(y)+\mathbb{M}_{6} \operatorname{dlog}(1+y) \\
& +\mathbb{M}_{7} \operatorname{dlog}(1-y)+\mathbb{M}_{8} \operatorname{dlog}\left(1+y^{2}\right) \\
& +\mathbb{M}_{9} \operatorname{dlog}(x+y)+\mathbb{M}_{10} \operatorname{dlog}(x-y) \\
& +\mathbb{M}_{11} \operatorname{dlog}(1+x y)+\mathbb{M}_{12} \operatorname{dlog}(1-x y)
\end{aligned}
$$

with $\mathbb{M}_{i} \in \mathbf{M}^{20 \times 20}(\mathbb{Q})$. Since all letters are algebraically rooted polynomials, we could derive the $\epsilon$-expansion of the general solution of Eq. (6) in terms of two-dimensional generalized polylogarithms (GPLs) [44-48], by iterative integration of the dlog-form, which we performed up $\mathcal{O}\left(\epsilon^{4}\right)$, i.e., to GPLs of weight four. In order to fully specify the analytic expression of the MIs, we complemented the general solution of DEs with a suitable set of boundary conditions. The latter were obtained by demanding the regularity of the MIs at the pseudothresholds $m_{H}^{2}=0$ and $m_{H}^{2}=4 m_{b}^{2}$, which appear as unphysical singularities of the DEs.

The expression of the MIs obtained in this way is valid in the Euclidean region $m_{H}^{2}<0 \wedge 0<m_{b}^{2}<m_{t}^{2}$, where the logarithms of Eq. (7) have no branch-cuts and, hence, the MIs are real. The values of the MIs for positive values of the Higgs squared momentum, and in particular for the decay region $m_{H}^{2}>4 m_{b}^{2}$, are obtained through analytic continuation, by propagating the Feynman prescription $m_{H}^{2} \rightarrow m_{H}^{2}+i 0^{+}$to the kinematic variables $x$ and $y$. All results have been numerically validated with GiNAC [49] against the results of SECDEC3 [50], both in the Euclidean and in the physical regions.

We note that the integrals defined in Eq. (3) appeared also in [51] in the context of $b b H$ production at the LHC, where, however, they were computed only in the large $m_{t}$ limit.

Upon inserting the expressions of the MIs into Eq. (2), we observed the expected analytic cancellation of all $\epsilon$-poles and obtained a finite result for $\left|\mathcal{M}_{y_{t}, b \bar{b}}\right|^{2}$,

$$
\left|\mathcal{M}_{y_{t}, b \bar{b}}\right|^{2}=\frac{\alpha_{s}^{2}}{\pi^{2}} C_{A} C_{F} y_{t} y_{b} m_{t} m_{b} \operatorname{Re} \mathcal{C}(x, y),
$$

with $\mathcal{C}(x, y)$ being a polynomial combination, with algebraic coefficients, of 256 distinct GPLs. The explicit expression of $\mathcal{C}(x, y)$, as well as of the newly computed MIs, can be released by the authors upon request.

\section{B. Real-virtual}

The real-virtual part of the computation involves the interference $\left|\mathcal{M}_{y_{t}, b \bar{b} g}\right|^{2}$ of the tree level amplitude for $H(q) \rightarrow b\left(p_{1}\right)+\bar{b}\left(p_{2}\right)+g\left(p_{3}\right)$ with the loop diagrams in Fig. 2 containing a closed top quark loop,

$$
\left|\mathcal{M}_{y_{t}, b \bar{b} g}\right|^{2} \equiv 2 \operatorname{Re} \mathcal{M}_{b \bar{b} g}^{(0) \dagger} \mathcal{M}_{y_{t}, b \bar{b} g}^{(1)} .
$$

We used standard techniques to evaluate the one loop amplitude. As expected, $\left|\mathcal{M}_{y_{t}, b \bar{b} g}\right|^{2}$ is finite (in $\epsilon$ ) and can be written as

$$
\begin{aligned}
\left|\mathcal{M}_{y_{t}, b \bar{b} g}\right|^{2}= & 32 \alpha_{s}^{2} C_{A} C_{F} y_{t} y_{b} m_{t} m_{b}\left(\frac{4\left(s_{12}+2 m_{b}^{2}\right)}{\left(s_{13}+s_{23}\right)^{2}}+\frac{s_{13}+s_{23}-4 m_{b}^{2}}{s_{13} s_{23}}\right) \\
& \times\left\{2\left[\sqrt{\frac{4 m_{t}^{2}}{m_{H}^{2}}-1}\left(\arctan \sqrt{\frac{4 m_{t}^{2}}{m_{H}^{2}}-1}-\frac{\pi}{2}\right)-\sqrt{\frac{4 m_{t}^{2}}{s_{12}+2 m_{b}^{2}}}-1\left(\arctan \sqrt{\frac{4 m_{t}^{2}}{s_{12}+2 m_{b}^{2}}-1}-\frac{\pi}{2}\right)\right]\right. \\
& \left.+\frac{s_{13}+s_{23}}{s_{12}+2 m_{b}^{2}}\left[1-\left(\frac{4 m_{t}^{2}}{s_{13}+s_{23}}-1\right)\left[\left(\arctan \sqrt{\frac{4 m_{t}^{2}}{m_{H}^{2}}-1}-\frac{\pi}{2}\right)^{2}-\left(\arctan \sqrt{\frac{4 m_{t}^{2}}{s_{12}+2 m_{b}^{2}}}-1-\frac{\pi}{2}\right)^{2}\right]\right]\right\},
\end{aligned}
$$


plus terms that vanish in four dimensions. In Eq. (10), $s_{i j}$ denotes twice the dot-product of momenta, $s_{i j} \equiv 2 p_{i} \cdot p_{j}$.

We integrated the real-virtual contribution over the whole phase space both analytically and numerically using Monte Carlo integration, finding perfect agreement. The analytic computation was performed by direct integration of $\left|\mathcal{M}_{y_{t}, b \bar{b} g}\right|^{2}$ over the three-particle phase space. The phase space measure for the decay $H(q) \rightarrow b\left(p_{1}\right)+\bar{b}\left(p_{2}\right)+$ $g\left(p_{3}\right)$ reads

$$
\begin{aligned}
\mathrm{d} P S_{3}= & 2^{-10+6 \epsilon} \pi^{-5+4 \epsilon}\left(q^{2}\right)^{-1+\epsilon}\left(\Delta_{3}\right)^{-\epsilon} \\
& \times \Theta\left(\Delta_{3}\right) \delta\left(q^{2}-2 m_{b}^{2}-s_{12}-s_{13}-s_{23}\right) \\
& \times \mathrm{d} \Omega_{d-1} \mathrm{~d} \Omega_{d-2} \mathrm{~d} s_{12} \mathrm{~d} s_{13} \mathrm{~d} s_{23},
\end{aligned}
$$

where $\Delta_{3}$ is given by

$$
\Delta_{3}=s_{12} s_{13} s_{23}-m_{b}^{2}\left(s_{13}^{2}+s_{23}^{2}\right) .
$$

The integral is finite in four dimensions and was evaluated in terms of GPLs after suitable transformations of the integration variables. In particular, square roots involving the integration variables appear at intermediate stages of the calculation (both from the one loop matrix element and from resolving the phase space constraint implied by the positivity of $\Delta_{3}$ ) and must be linearized, e.g., by using the techniques of [52]. The full result is represented in terms of a formula with 1841 distinct GPLs and can be released by the authors upon request.

The numerical integration of the real-virtual contribution is straightforward and has been used to validate the analytic computation. It also allows us to build Monte Carlo simulations with acceptance cuts and has been used to obtain the differential result of the next section.

\section{RESULTS}

We begin the presentation of our results by discussing the inclusive decay rate. In Table I, we compare the exact formula, obtained from the sum of the double virtual and real-virtual contributions described in the previous section, to the approximated one of Ref. [18]. Apart from an overall mass dependence which fixes the dimension of the decay rate, the result depends on two ratios of masses. Thus we find it the cleanest to perform this comparison by varying

TABLE I. The discrepancy $\mathbf{d}$ between our result and the approximate formula in [18], we fix $m_{b}=2.8 \mathrm{GeV}$.

\begin{tabular}{ccccc}
\hline \hline & \multicolumn{5}{c}{$m_{H}$} \\
\hline$m_{t}$ & 20 & 75 & 125 & 180 \\
\hline 100 & 0.125 & 0.131 & 1.254 & 7.424 \\
125 & 0.133 & 0.052 & 0.479 & 2.475 \\
175 & 0.143 & 0.013 & 0.117 & 0.566 \\
250 & 0.152 & 0.003 & 0.027 & 0.127 \\
350 & 0.159 & 0.001 & 0.007 & 0.032 \\
\hline \hline
\end{tabular}

two of the mass parameters, while keeping the third, which we choose to be the bottom quark mass, fixed. Since the approximated result of Ref. [18] involves the running bottom quark mass, we have chosen to fix $m_{b}=2.8 \mathrm{GeV}$ in Table I, which corresponds approximately to $m_{b}\left(m_{H}\right)$ for the physical Higgs mass and bottom quark pole mass. The numbers in the table are obtained with the following formula for the relative discrepancy among exact and approximated results:

$$
\mathbf{d}=100\left(1-\frac{\Gamma_{y_{t}}^{\text {Approx }}}{\Gamma_{y_{t}}^{\text {Exact }}}\right) \text {. }
$$

The agreement is excellent for the physical mass values, proving for the first time and in a completely independent way the validity of the approximated formula. The fact that the approximated formula of Ref. [18] works at least an order of magnitude better then expected strengthens the validity of other results obtained through similar expansions and that still cannot be evaluated analytically.

We now turn to the second question regarding the impact of the $y_{t}$ contribution at differential level. To this extent, we present results for Higgs boson associated production in $13 \mathrm{TeV}$ proton-proton collisions and, in order to avoid the contamination from initial state radiation, we consider $p p \rightarrow$ $W^{+}\left(l^{+} \nu_{l}\right) H(b \bar{b})$ at leading order and add the corrections to the decay process at the next-to-leading order. Then, we compare this result with the one obtained by adding also the $y_{t}$ contribution. Note that in both cases we normalize the cross section to the total Higgs boson decay width into bottom quarks reported in the Yellow Report of the Higgs Cross Section Working Group [53] (HXSWG) and computed with the code HDECAY [54-56], that includes all the higher order corrections mentioned in the Introduction. In particular, we set $m_{H}=125 \mathrm{GeV}$ and $\Gamma_{H \text {,tot }}=4.131 \mathrm{MeV}$, while $\operatorname{Br}(H \rightarrow b \bar{b})=0.5824$. We define the baseline prediction (NLO) and the prediction including the $y_{t}$ contribution $\left(\mathrm{NLO}+y_{t}\right)$ by the formulas:

$$
\begin{aligned}
d \sigma_{h_{1} h_{2} \rightarrow W H \rightarrow W b \bar{b}}^{\mathrm{NLO}}= & d \sigma_{h_{1} h_{2} \rightarrow W H}^{\mathrm{LO}} \times \frac{d \Gamma_{H \rightarrow b \bar{b}}^{\mathrm{NLO}}}{\Gamma_{H \rightarrow b \bar{b}}^{\mathrm{NLO}}} \times \operatorname{Br}(H \rightarrow b \bar{b}), \\
d \sigma_{h_{1} h_{2} \rightarrow W H \rightarrow W b \bar{b}}^{\mathrm{NLO}+y_{t}}= & d \sigma_{h_{1} h_{2} \rightarrow W H}^{\mathrm{LO}} \times \frac{d \Gamma_{H \rightarrow b \bar{b}}^{\mathrm{NLO}}+d \Gamma_{H \rightarrow b \bar{b}}^{y_{t}}}{\Gamma_{H \rightarrow b \bar{b}}^{\mathrm{NLO}}+\Gamma_{H \rightarrow b \bar{b}}^{y_{t}}} \\
& \times \operatorname{Br}(H \rightarrow b \bar{b}),
\end{aligned}
$$

where $d \Gamma_{H \rightarrow b \bar{b}}^{\mathrm{NLO}}$ and $d \Gamma_{H \rightarrow b \bar{b}}^{y_{t}}$ include both the virtual and real emission corrections and the counterterms for the soft radiation, to be integrated over their respective phase spaces. Clearly, for observables that are inclusive over the Higgs boson decay products, both Eqs. (14) and (15) yield the leading order production cross section times the branching ratio, so effectively we are comparing the shapes of distributions. We use the SM parameters recommended by the 


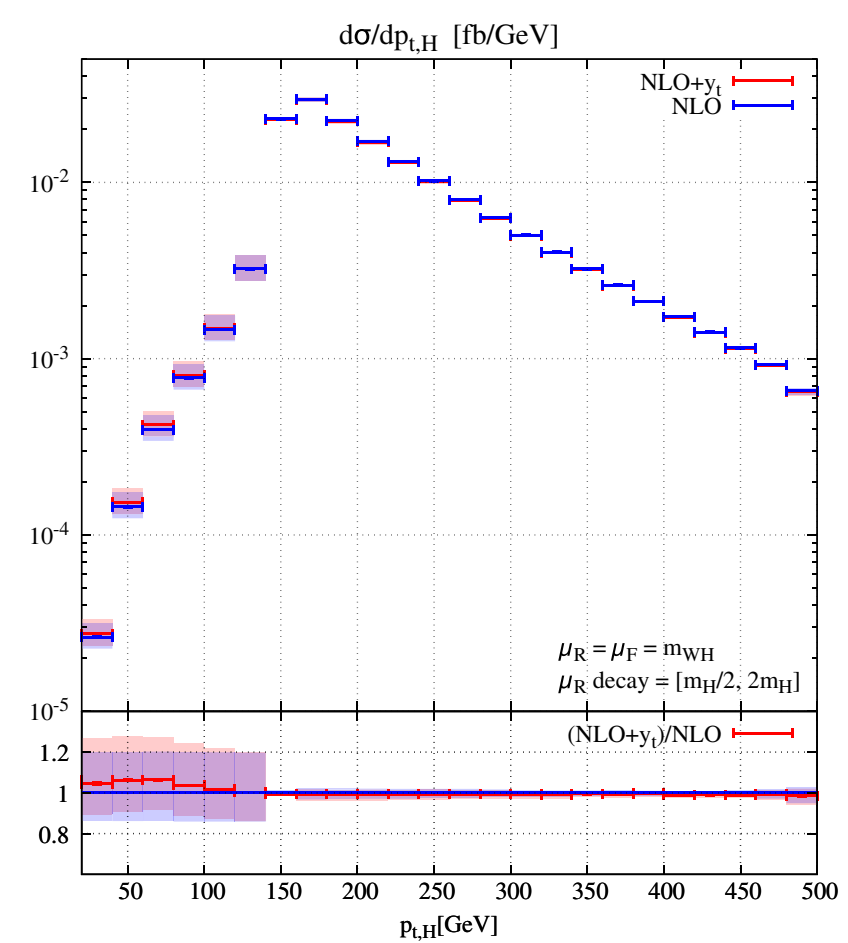

FIG. 3. Transverse momentum distribution of the two b-jet system from $W H(b b)$ production in proton proton collisions at $14 \mathrm{TeV}$. Only corrections to the Higgs decay into bottom quarks are included.

HXSWG and the NNPDF30_nnlo_as_0118 parton distribution functions [57]. In particular, we consider decoupled top quark with on shell mass $m_{t}=172.5 \mathrm{GeV}$. As for the bottom quark, we compute values of the bottom Yukawa coupling for a given renormalization scale assuming $m_{b}\left(m_{b}\right)=4.18 \mathrm{GeV}$ and two loop running, while we use the corresponding on shell mass $m_{b}=4.92 \mathrm{GeV}$ for the kinematics. Note that in the $\left(\mathrm{NLO}+y_{t}\right)$ cross section we combine $y_{b}^{2}$ and $y_{b} y_{t}$ terms so that, despite cross section normalization, the actual value of $y_{b}$ is relevant. Furthermore, we impose the following typical lepton acceptance cuts: selected events must have a missing transverse momentum greater than $30 \mathrm{GeV}$, the charged lepton is required to have a transverse momentum greater than $15 \mathrm{GeV}$ and an absolute rapidity smaller than 2.5 and, finally, the $\mathrm{W}$ boson transverse momentum is required to be larger than $150 \mathrm{GeV}$. We reconstruct jets using the anti-kt algorithm with the resolution variable set to 0.5 and require two b-jets with transverse momentum greater than $25 \mathrm{GeV}$ and absolute rapidity smaller than 2.5 .

In Figs. 3 and 4 we present the transverse momentum and mass distributions of the two b-jet system. The error bars, barely visible in the figures, represent the statistical uncertainty associated with Monte Carlo integration while the bands are obtained by varying the renormalization scale between $m_{H} / 2$ and $2 m_{H}$. We observe that the impact of the $y_{t}$ contribution on both distributions is quite small, adding

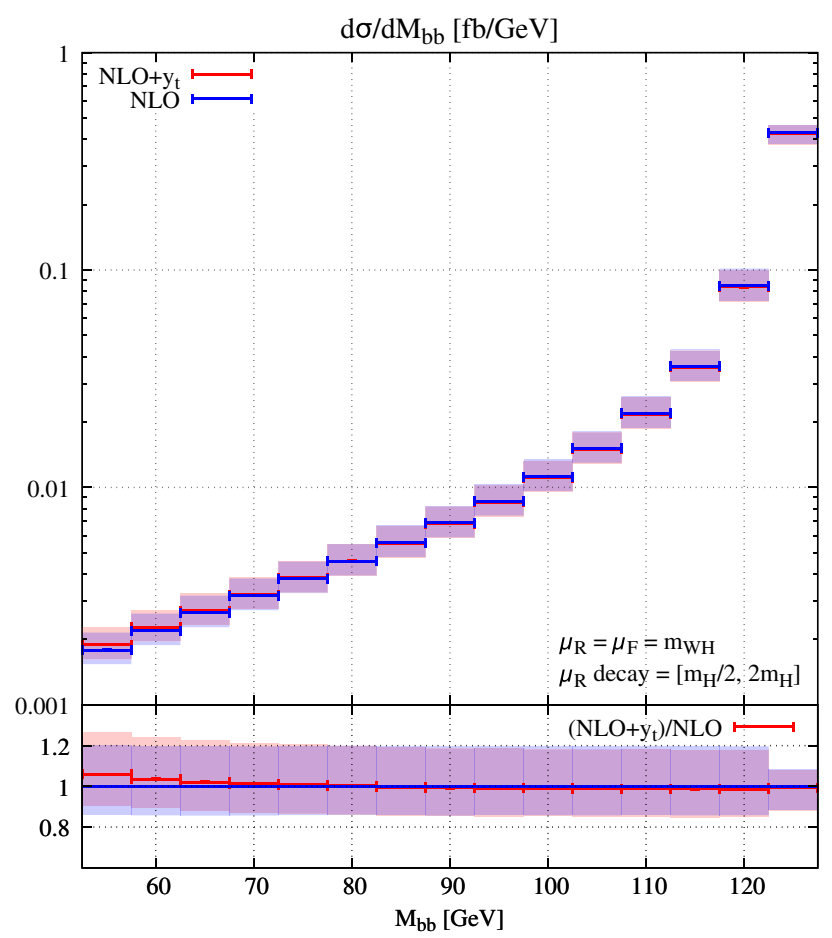

FIG. 4. Mass distribution of the two b-jet system from $W H(b b)$ production in proton proton collisions at $14 \mathrm{TeV}$. Only corrections to the Higgs decay into bottom quarks are included.

a $\sim 5 \%$ effect only in the low energy tail of each. We mention that, by using identical cuts, Ref. [27] finds that the NNLO corrections (not including $y_{t}$ contributions) to Higgs decay also enhance the low energy region of the $p_{t, H}$ distribution by a similar, though somewhat lower amount. The situation is rather different for the invariant mass distribution, where the NNLO corrections considered in [27] produce a large increase in the low mass region.

Finally, we note that the size of the $y_{t}$ contribution is generally much smaller than the scale variation uncertainty of the computation, except over the high energy region of the transverse momentum distribution, where the scale uncertainty is itself small. This can be understood by noticing that the leading order calculation would produce an invariant mass distribution which exactly fulfills the constraint $M_{b \bar{b}}=m_{H}$ and a transverse momentum distribution with $p_{t, H}>150 \mathrm{GeV}$ to compensate the required minimal transverse momentum of the $W$ boson. Hence, the NLO prediction for the $M_{b \bar{b}}$ distribution, as well as the low energy region of the $p_{t, H}$ distribution, is effectively a leading order calculation. In contrast, the high energy region of the $p_{t, H}$ distribution corresponds to a genuine NLO calculation, which is reflected in the much reduced renormalization scale uncertainty.

\section{CONCLUSIONS}

In this paper we presented the results of the full analytic computation of the top Yukawa contribution to the Higgs 
boson decay width into bottom quarks. First, we demonstrated that the approximate formula used so far in the literature works exceedingly well for physical values of the masses. This nice behavior was not predictable a priori and, with respect to a possible error estimate ranging from $2 \%$ to $20 \%$ for the physical mass values, we have instead found per mille level deviations of this formula from the exact result. This better than expected behavior also strengthens the validity of other approximated results obtained through mass expansions and deserves further investigation. Furthermore, we showed that the impact of the $y_{t}$ contribution at the differential level is quite small so that Monte Carlo simulations of associated $V H$ production with $H \rightarrow b \bar{b}$ decay are not affected by an additional significant source of uncertainty due to the neglected terms proportional to $y_{t}$.

\section{ACKNOWLEDGMENTS}

This work has been supported by the Swiss National Science Foundation under Grant No. 200020-175595 (AP), the Italian Ministry of Education and Research MIUR, under Project No. 2015P5SBHT, by the INFN Iniziativa Specifica ENP (FT) and by Grant No. K 125105 of the National Research, Development and Innovation Fund in Hungary (GS).
[1] P. W. Higgs, Phys. Lett. 12, 132 (1964).

[2] F. Englert and R. Brout, Phys. Rev. Lett. 13, 321 (1964);

[3] G. Aad et al. (ATLAS Collaboration), Phys. Lett. B 716, 1 (2012).

[4] S. Chatrchyan et al. (CMS Collaboration), Phys. Lett. B 716, 30 (2012).

[5] J. P. Miller, E. de Rafael, B. L. Roberts, and D. Stckinger, Annu. Rev. Nucl. Part. Sci. 62, 237 (2012).

[6] E. Graverini (ATLAS, CMS, and LHCb Collaborations), arXiv:1807.11373.

[7] M. Aaboud et al. (ATLAS Collaboration), Phys. Lett. B 784, 173 (2018).

[8] A. M. Sirunyan et al. (CMS Collaboration), J. High Energy Phys. 08 (2018) 066.

[9] A. M. Sirunyan et al. (CMS Collaboration), Phys. Lett. B 779, 283 (2018).

[10] M. Aaboud et al. (ATLAS Collaboration), arXiv:1811 .08856 [Phys. Rev. (to be published)].

[11] M. Aaboud et al. (ATLAS Collaboration), Phys. Lett. B 786, 59 (2018).

[12] A. M. Sirunyan et al. (CMS Collaboration), Phys. Rev. Lett. 121, 121801 (2018).

[13] S. G. Gorishnii, A. L. Kataev, S. A. Larin, and L. R. Surguladze, Mod. Phys. Lett. A 05, 2703 (1990).

[14] S. G. Gorishnii, A. L. Kataev, S. A. Larin, and L. R. Surguladze, Phys. Rev. D 43, 1633 (1991).

[15] A. L. Kataev and V. T. Kim, Mod. Phys. Lett. A 09, 1309 (1994).

[16] L. R. Surguladze, Phys. Lett. B 341, 60 (1994).

[17] S. A. Larin, T. van Ritbergen, and J. A. M. Vermaseren, Phys. Lett. B 362, 134 (1995).

[18] K. G. Chetyrkin and A. Kwiatkowski, Nucl. Phys. B461, 3 (1996).

[19] K. G. Chetyrkin, Phys. Lett. B 390, 309 (1997).

[20] P. A. Baikov, K. G. Chetyrkin, and J. H. Kuhn, Phys. Rev. Lett. 96, 012003 (2006).

[21] A. Dabelstein and W. Hollik, Z. Phys. C 53, 507 (1992).

[22] B. A. Kniehl, Nucl. Phys. B376, 3 (1992).

[23] A. L. Kataev, JETP Lett. 66, 327 (1997).
[24] L. Mihaila, B. Schmidt, and M. Steinhauser, Phys. Lett. B 751, 442 (2015).

[25] W. Bernreuther, L. Chen, and Z.-G. Si, J. High Energy Phys. 07 (2018) 159.

[26] B. A. Kniehl, Phys. Lett. B 343, 299 (1995).

[27] G. Ferrera, G. Somogyi, and F. Tramontano, Phys. Lett. B 780, 346 (2018).

[28] F. Caola, G. Luisoni, K. Melnikov, and R. Rntsch, Phys. Rev. D 97, 074022 (2018).

[29] A. Kotikov, Phys. Lett. B 254, 158 (1991).

[30] E. Remiddi, Nuovo Cimento A 110, 1435 (1997).

[31] T. Gehrmann and E. Remiddi, Nucl. Phys. B580, 485 (2000).

[32] M. Argeri and P. Mastrolia, Int. J. Mod. Phys. A 22, 4375 (2007).

[33] F. V. Tkachov, Phys. Lett. 100B, 65 (1981).

[34] K. Chetyrkin and F. Tkachov, Nucl. Phys. B192, 159 (1981).

[35] S. Laporta, Int. J. Mod. Phys. A 15, 5087 (2000).

[36] A. von Manteuffel and C. Studerus, arXiv:1201.4330.

[37] M. Argeri, S. Di Vita, P. Mastrolia, E. Mirabella, J. Schlenk, U. Schubert, and L. Tancredi, J. High Energy Phys. 03 (2014) 082.

[38] S. Di Vita, P. Mastrolia, U. Schubert, and V. Yundin, J. High Energy Phys. 09 (2014) 148.

[39] R. Bonciani, S. Di Vita, P. Mastrolia, and U. Schubert, J. High Energy Phys. 09 (2016) 091.

[40] S. Di Vita, P. Mastrolia, A. Primo, and U. Schubert, J. High Energy Phys. 04 (2017) 008.

[41] P. Mastrolia, M. Passera, A. Primo, and U. Schubert, J. High Energy Phys. 11 (2017) 198.

[42] S. Di Vita, S. Laporta, P. Mastrolia, A. Primo, and U. Schubert, J. High Energy Phys. 09 (2018) 016.

[43] J. M. Henn, Phys. Rev. Lett. 110, 251601 (2013).

[44] A. Goncharov, Proc. Int. Cong. Math. 1-2, 374 (1995).

[45] E. Remiddi and J. Vermaseren, Int. J. Mod. Phys. A 15, 725 (2000).

[46] T. Gehrmann and E. Remiddi, Comput. Phys. Commun. 141, 296 (2001). 
[47] T. Gehrmann and E. Remiddi, Comput. Phys. Commun. 144, 200 (2002).

[48] J. Vollinga and S. Weinzierl, Comput. Phys. Commun. 167, 177 (2005).

[49] C. W. Bauer, A. Frink, and R. Kreckel, arXiv:cs/0004015.

[50] S. Borowka, G. Heinrich, S. P. Jones, M. Kerner, J. Schlenk, and T. Zirke, Comput. Phys. Commun. 196, 470 (2015).

[51] N. Deutschmann, F. Maltoni, M. Wiesemann, and M. Zaro, arXiv:1808.01660 [J. High Energy Phys. (to be published)].

[52] M. Besier, D. Van Straten, and S. Weinzierl, arXiv:1809.10983.
[53] D. de Florian et al. (LHC Higgs Cross Section Working Group), arXiv:1610.07922.

[54] A. Djouadi, J. Kalinowski, and M. Spira, Comput. Phys. Commun. 108, 56 (1998).

[55] M. Spira, Fortschr. Phys. 46, 203 (1998).

[56] A. Djouadi, M. M. Muhlleitner, and M. Spira, Acta Phys. Pol. B 38, 635 (2007).

[57] R. D. Ball et al. (NNPDF Collaboration), J. High Energy Phys. 04 (2015) 040. 